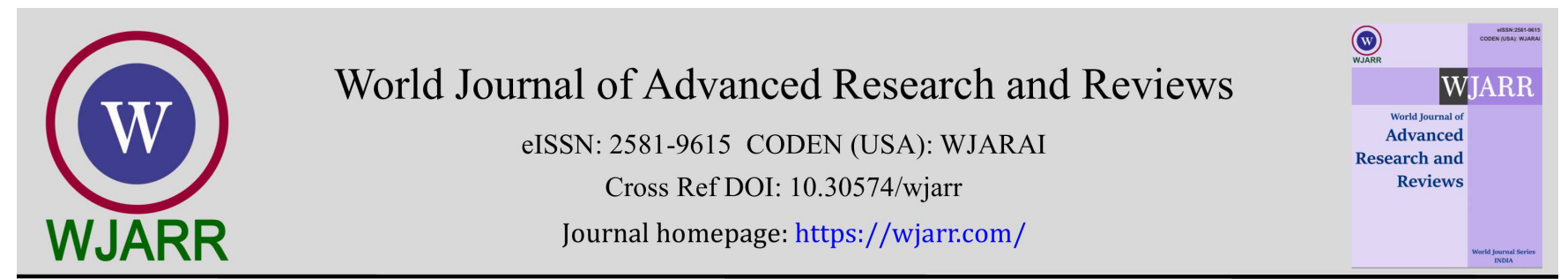

(REVIEW ARTICLE)

Check for updates

\title{
Understanding childhood immunizations, their serological interpretation and vaccines - A review article
}

\author{
Andrew Kiboneka * \\ Department of Paediatrics, Case Hospital, Kampala, Uganda.
}

World Journal of Advanced Research and Reviews, 2021, 10(03), 233-238

Publication history: Received on 05 May 2021; revised on 06 June 2021; accepted on 09 June 2021

Article DOI: https://doi.org/10.30574/wjarr.2021.10.3.0275

\begin{abstract}
The practice of immunization dates back hundreds of years. Buddhist monks drank snake venom to confer immunity to snake bite and variolation (smearing of a skin tear with cowpox to confer immunity to smallpox) was practiced in $17^{\text {th }}$ century China.

Edward Jenner is considered the founder of vaccinology in the West in 1796, after he inoculated an 8 year-old-boy with vaccinia virus (cowpox), and demonstrated immunity to smallpox. In 1798, the first smallpox vaccine was developed. Over the $18^{\text {th }}$ and $19^{\text {th }}$ centuries, systematic implementation of mass smallpox immunization culminated in its global eradication in 1979. Vaccination is when a vaccine is administered to you (usually by injection). Immunization is what happens in your body after you have the vaccination.

The vaccine stimulates your immune system so that it can recognize the disease and protect you from future infection (i.e., you become immune to the infection.). Immunization is a proven tool for controlling and eliminating lifethreatening infectious diseases and is estimated to avert between 2 and 3 million deaths each year. It is one of the most cost-effective health investments, with proven strategies that make it accessible to even the most hard-to-reach and vulnerable populations. It has clearly defined target groups; it can be delivered effectively through outreach activities; and vaccination does not require any major lifestyle change.
\end{abstract}

Keywords: Immunizations; Vaccinations; Childhood illnesses; Hepatitis A and B

\section{Methodology}

A literature search review on PubMed Search engine. All articles between January-2015 through May-2021. Search items included Immunizations, Vaccinations and Childhood diseases.

\section{Results}

According to the World Health Organization (WHO), "immunization is the process whereby a person is made immune or resistant to an infectious disease, typically by the administration of a vaccine [1]. Vaccines stimulate the body's own immune system to protect the person against subsequent infection or disease" [2].

A person becomes immune to a disease when the body has been exposed to it either through illness or vaccination. The immune system develops antibodies to the disease so that it cannot make you sick again -Vaccine-preventable diseases:

\footnotetext{
* Corresponding author: Andrew Kiboneka

Department of Paediatrics Case Hospital, Kampala, Uganda.

Copyright (C) 2021 Author(s) retain the copyright of this article. This article is published under the terms of the Creative Commons Attribution Liscense 4.0.
} 
A vaccine-preventable disease is an infectious disease for which an effective preventive vaccine exists. If a person acquires a vaccine-preventable disease and dies from it, the death is considered a vaccine-preventable death [1].

The most common and serious vaccine-preventable diseases tracked by the World Health Organization (WHO) are: diphtheria, Haemophilus influenza serotype $b$ infection, hepatitis $B$, measles, meningitis, mumps, pertussis, poliomyelitis, rubella, tetanus, tuberculosis, and yellow fever. The WHO reports licensed vaccines being available to prevent, or contribute to the prevention and control of, 25 vaccine-preventable infections [1].

\section{Types of vaccines}

Scientific research has led to the development of numerous types of vaccines that safely elicit immune responses that protect against infection, and researchers continue to investigate novel vaccine strategies for prevention of existing and emerging infectious diseases. Recent decades have brought major advances in understanding the complex interactions between the microbes that cause disease and their human hosts. These insights, as well as advances in laboratory techniques and technologies, have aided the development of new types of vaccines There are many approaches to vaccine development, but vaccines can be broadly classified by how the antigen(s), the active component(s) that generate a specific immune response against the disease-causing organism, are prepared.

There are 4 main types of vaccines

- $\quad$ Live-attenuated vaccines

- Inactivated vaccines

- Subunit, recombinant, polysaccharide, and conjugate vaccines

- Toxoid vaccines.

In addition, we have DNA and recombinant vaccines

NB. DNA vaccines are easy and inexpensive to make-and they produce strong, long-term immunity.

- Recombinant vector vaccines (platform-based vaccines) act like a natural infection, so they're especially good at teaching the immune system how to fight germs [3].

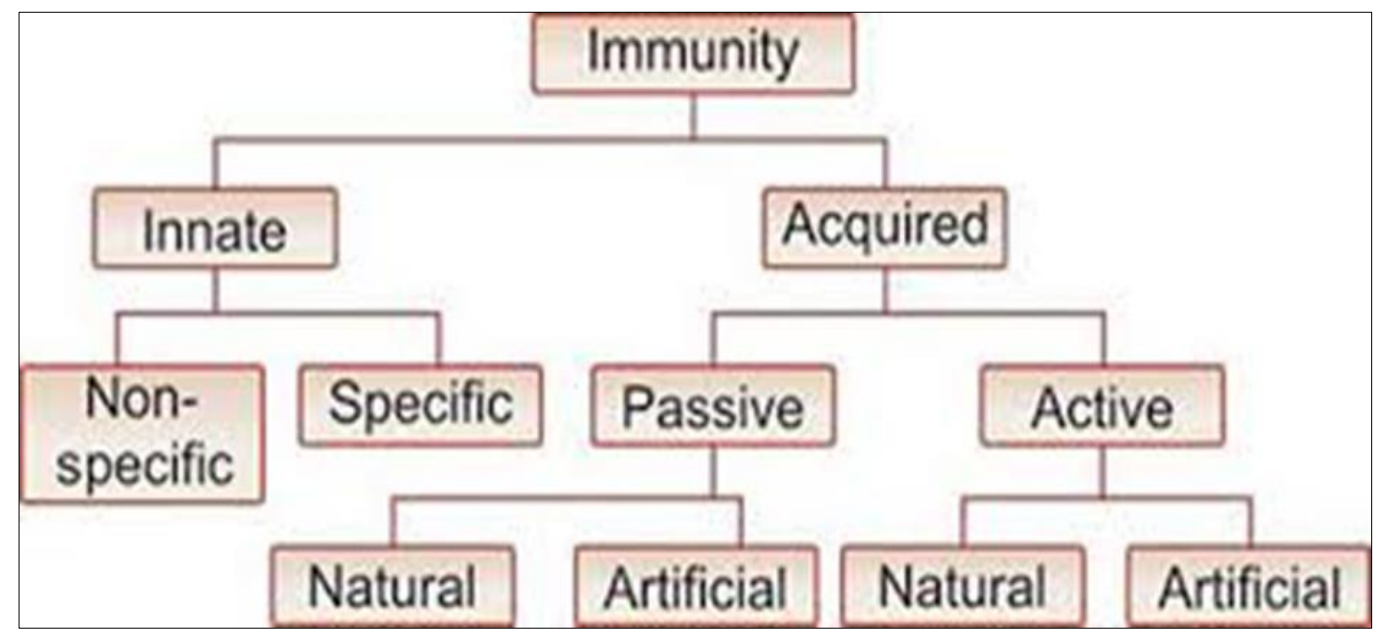

Figure 1 Classification of Immunity

The ability of an organism to resist disease, either through the activities of specialized blood cells or antibodies produced by them in response to natural exposure or inoculation (active immunity) or by the injection of antiserum or the transfer of antibodies from a mother to her baby via the placenta or breast milk (passive immunity).

\subsection{Natural versus acquired Immunity}

Natural immunity occurs through contact with a disease-causing agent, when the contact was not deliberate, whereas artificial immunity develops only through deliberate actions of exposure. Immunity is the state of protection against 
infectious disease conferred either through an immune response generated by immunization or previous infection, or by other non-immunological factors.

There are two ways to acquire active resistance against invading microbes: active natural and active artificial.

\section{Vaccination}

Vaccination is the most effective method of preventing infectious diseases; widespread immunity due to vaccination is largely responsible for the worldwide eradication of smallpox and the curbing of diseases such as polio, measles, and tetanus from much of the world.

\subsection{Vaccine Adjuvants}

- Efforts to develop safe and effective vaccines increasingly involve the use of adjuvants-substances formulated as part of a vaccine to boost immune responses and enhance the vaccine's effectiveness.

- Vaccine adjuvants accelerate, enhance and prolong the immune responses triggered by antigens-the vaccine components that elicit pathogen-specific immune responses.

Certain populations, such as people with compromised immune systems, the elderly and the very young, particularly benefit from vaccines with adjuvants because their immune systems may require an extra boost to provide protection. Adjuvants also can allow vaccine developers to use less antigen, which in some cases may be in short supply or costly.

Additionally, adjuvant vaccines can elicit more durable immune responses, reducing or eliminating the need for booster vaccinations. Aluminum-containing adjuvants, collectively termed alum, have been safely used in vaccines since the 1930s and are still widely used today. Aluminum is among the most common metals found in nature and is present in food and water [4].

Scientific research has shown that the trace amounts of aluminum in vaccines are safe and not readily absorbed by the body. For many years, alum was the only adjuvant added to vaccines in the United States.

In recent decades, however, technologic and scientific advances have increased understanding of human immunity, and these insights have led to the identification of new adjuvants and many promising adjuvant candidates.

In 2009, FDA approved Cervarix, a human papillomavirus vaccine containing a novel adjuvant called AS04. Since then, several additional vaccines containing novel adjuvants have been approved for use in the United States.

\subsection{Immunity}

Immunity is defined as the body's ability to protect itself from an infectious disease. When you are immune to a disease, your immune system can fight off infection from it.

Immunity is either innate or adaptive. Innate immunity, also known as natural or genetic immunity, is immunity that an organism is born with. This type of immunity is encoded in one's genes. Genetic immunity protects an organism throughout their entire life. Innate immunity consists of:

\section{- External defenses}

Known as the first line of defense, external defenses work to protect an organism from pathogen exposure. External defenses include things like the skin, tears, and stomach acid.

\section{- Internal defenses}

Known as the second line of defense, internal defenses address a pathogen once it has entered the body. Internal defenses include things like inflammation and fevers [5].

\subsection{Hepatitis}

Hepatitis is an inflammation of the liver. The condition can be self-limiting or can progress to fibrosis (scarring), cirrhosis or liver cancer. Hepatitis viruses are the most common cause of hepatitis in the world but other infections, toxic substances (e.g. alcohol, certain drugs), and autoimmune diseases can also cause hepatitis [3]. 
There are 5 main hepatitis viruses, referred to as types A, B, C, D and E. These 5 types are of greatest concern because of the burden of illness and death they cause and the potential for outbreaks and epidemic spread. In particular, types $\mathrm{B}$ and $\mathrm{C}$ lead to chronic disease in hundreds of millions of people and, together, are the most common cause of liver cirrhosis and cancer [3].

\subsection{Hepatitis B}

Although a vaccine to prevent hepatitis B infection is available, hepatitis B-induced liver cirrhosis and liver cancer kill about 3,000 people in the United States and roughly 620,000 people worldwide each year.

- The virus can be spread

- From mother to child during childbirth

- Through sex with an infected partner

- Through contact with the blood of an infected person

- By sharing needles, syringes, razors, or toothbrushes with an infected person

Co-infection with hepatitis B virus and HIV is common.

Table 1 Hepatitis B Serology Interpretation

\begin{tabular}{|c|c|c|}
\hline $\begin{array}{l}\text { HBsAg } \\
\text { Anti-HBc } \\
\text { Anti--HBs }\end{array}$ & $\begin{array}{l}\text { negative } \\
\text { negative } \\
\text { negative }\end{array}$ & susceptible \\
\hline $\begin{array}{l}\text { HBsAg } \\
\text { Anti-HBc } \\
\text { Anti--HBs }\end{array}$ & $\begin{array}{l}\text { negative } \\
\text { positive } \\
\text { positive }\end{array}$ & Immune due to natural infection \\
\hline $\begin{array}{l}\text { HBsAg } \\
\text { Anti-HBc } \\
\text { Anti--HBs }\end{array}$ & $\begin{array}{l}\text { negative } \\
\text { negative } \\
\text { positive }\end{array}$ & Immune due to hepatitis B vaccination \\
\hline $\begin{array}{l}\text { HBsAg } \\
\text { Anti--HBc } \\
\text { IgM anti-HBc } \\
\text { Anti-HBs }\end{array}$ & $\begin{array}{l}\text { positive } \\
\text { positive } \\
\text { positive } \\
\text { negative }\end{array}$ & Acutely infected \\
\hline $\begin{array}{l}\text { HBsAg } \\
\text { Anti--HBc } \\
\text { IgM anti-HBc } \\
\text { Anti-HBs }\end{array}$ & $\begin{array}{l}\text { positive } \\
\text { positive } \\
\text { negative } \\
\text { negative }\end{array}$ & Chronically infected \\
\hline $\begin{array}{l}\text { HBsAg } \\
\text { Anti--HBc } \\
\text { Anti-HBs }\end{array}$ & $\begin{array}{l}\text { negative } \\
\text { positive } \\
\text { negative }\end{array}$ & $\begin{array}{l}\text { Interpretation unclear: four possibilities: } \\
\text { Resolved infection (most common) } \\
\text { False-positive anti-HBc, thus susceptible } \\
\text { "low level" chronic infection } \\
\text { Resolving acute infection }\end{array}$ \\
\hline
\end{tabular}

\subsection{Hepatitis B vaccination of infants, children, and adolescents}

The Advisory Committee on Immunization Practices (ACIP) recommends: 
- Administration of hepatitis B vaccine and hepatitis B immune globulin (HBIG) for infants born to HBV-infected women within 12 hours of birth, followed by completion of the vaccine series and post vaccination serologic testing;

- Universal hepatitis B vaccination within 24 hours of birth, followed by completion of the vaccine series; and

- Vaccination of children and adolescents aged $<19$ years who have not been vaccinated previously [6].

Hepatitis B vaccine is produced by recombinant DNA technology, most commonly in yeast.

The complete vaccination series consists of three doses of vaccine; the first two doses are usually given 1 month apart, with the third dose 1-12 months later.

\subsection{Hepatitis A}

Hepatitis A vaccine (Havrix, Vaqta) is used to prevent hepatitis A, a type of liver disease that is caused by the hepatitis A virus (HAV). Hepatitis A is usually spread when a person ingests fecal matter from contact with food, drinks, or objects which have been contaminated by feces or stool of an HAV-infected person. Hepatitis A infection can be mild with no symptoms or a serious illness that can rarely cause liver failure and death. Getting vaccinated against the hepatitis $\mathrm{A}$ virus is the best way to prevent these problems.

The hepatitis A vaccine is used for the prevention of disease caused by hepatitis A virus in persons 12 months of age and older. The primary dose should be given at least 2 weeks prior to expect exposure to HAV. Hepatitis A vaccine was approved in 2005. Hepatitis A vaccine is a vaccine that prevents hepatitis A.

It is effective in around 95\% of cases and lasts for at least fifteen years and possibly a person's entire life. If given, two doses are recommended beginning after the age of one. It is given by injection into a muscle.

\subsection{Diphtheria and tetanus}

- Tdap is a combination vaccine that protects against three potentially life-threatening bacterial diseases: tetanus, diphtheria, and pertussis (whooping cough).

- $\mathrm{Td}$ is a booster vaccine for tetanus and diphtheria.

- It does not protect against pertussis.

- Tetanus enters the body through a wound or cut.

- Children younger than 7 years of age receive DTaP or DT, while older children and adults receive Tdap and Td.

- Give infants and children 5 doses of DTaP.

- Give one dose at each of these ages: 2 months, 4 months, 6 months, 15 through 18 months, and 4 through 6 years.

- Use DT for infants and children who should not receive acellular pertussis-containing vaccines.

- Give adolescents a single dose of Tdap, preferably at 11 to 12 years of age.

\subsection{Catch up immunization schedule for tetanus}

- Adolescents age 13-18 years who have not received Tdap:

- 1 dose Tdap, then Td or Tdap booster every 10 years.

- Persons age 7-18 years not fully vaccinated* with DTaP:

- 1 dose Tdap as part of the catch-up series (preferably the first dose); if additional doses are needed, use Td or Tdap.

- Tdap administered at 7-10 years.

\subsection{Chickenpox}

Chickenpox is a highly contagious disease caused by the varicella-zoster virus (VZV). It can cause an itchy, blister-like rash. The rash first appears on the chest, back, and face, and then spreads over the entire body, causing between 250 and 500 itchy blisters. Chickenpox can be serious, especially in babies, adolescents, adults, pregnant women, and people with bodies that have a lowered ability to fight germs and sickness (weakened immune system). The best way to prevent chickenpox is to get the chickenpox vaccine [7]. 


\subsection{Tuberculosis}

- It was hoped that with the invention of the BCG vaccine and medicines, it would be possible to wipe out TB in the same way that smallpox has been eradicated.

- This has turned out to be difficult because Much of the initial improvement in TB rates in more developed countries was related to improvements in housing, nutrition and access to treatment, but these issues are still present in many countries that are less developed

- Several strains of TB bacteria have developed resistance to 1 or more anti-TB medications, making them much harder to treat

- The BCG vaccination is effective against severe forms of the disease, such as TB meningitis in children, but it's not as effective against all forms of TB [8].

\section{Conclusion}

Immunization is the process whereby a person is made immune or resistant to an infection, typically by the administration of a vaccine. Vaccines stimulate the body's own immune system to protect the person against subsequent infection. Immunizations protect us from serious diseases and also prevent the spread of those diseases to others. Over the year's immunizations have thwarted epidemics of once common infectious diseases such as measles, mumps, and whooping cough. The WHO defines immunization as "the process whereby a person is made immune or resistant to an infectious disease, typically by the administration of a vaccine.

\section{Compliance with ethical standards}

\section{Acknowledgments}

Professor Peter Nyarango of the University of Namibia and Professor Phillipa Musoke of Makerere University for their mentorship

\section{References}

[1] World Health Organization

[2] Center for Disease Control and Prevention

[3] Red book. Report of the Committee on Infectious Diseases of the American Academy of Pediatrics.2021

[4] Pulendran, B., S. Arunachalam, P. \& O'Hagan, D.T. Emerging concepts in the science of vaccine adjuvants. Nat Rev Drug Discov 20, 454-475 (2021). https://doi.org/10.1038/s41573-021-00163-y.

[5] Jean S. Marshall, Richard Warrington, Wade Watson, Harold L. Kim. An introduction to immunology and immunopathology. Allergy Asthma Clin Immunol. 2018; 14(Suppl 2): 49. Published online 2018 Sep 12. doi: $10.1186 / \mathrm{s} 13223-018-0278-1$

[6] The Advisory Committee on Immunization Practices. Centers for Disease Control and Prevention

[7] Gershon, A., Breuer, J., Cohen, J. et al. Varicella zoster virus infection. Nat Rev Dis Primers 1, 15016 (2015). https://doi.org/10.1038/nrdp.2015.16

[8] Robindra Basu Roy, Elizabeth Whittaker, James A Seddon, Professor Beate Kampmann . Children and Mycobacterium tuberculosis: a review of susceptibility and protection. Lancet Infect Dis. 2019 Mar; 19(3): e96e108 